\title{
Imaging the Structure and Properties of 2D Materials with 4D-STEM
}

\author{
David Muller $^{1}$, Zhen Chen ${ }^{1}$, Yi Jiang ${ }^{2}$ and Michal Odstrcil ${ }^{3}$
}

${ }^{1}$ Cornell University, Ithaca, New York, United States, ${ }^{2}$ Argonne National Laboratory, Lemont, Illinois, United States, ${ }^{3}$ Paul Scherrer Institut, Villigen PSI, Aargau, Switzerland

Two-dimensional materials are usually imaged with low beam energies to minimize sample damage but limiting spatial resolution to $\sim 1 \AA$. With the recent advances in detector technology [1] and reconstruction algorithms [2], the resolution limits are now dominated by counting noise through the maximum allowable dose, either by radiation damage to the sample, or by recording times. In particular, ptychographic phase retrieval algorithms offer an approach to using all of the scattered electrons - potentially enhancing both the resolution and dose-efficiency beyond what is possible with conventional imaging modes. The intrinsically thin nature of 2D materials greatly aids the modelling of the scattering process needed for phase retrieval. We have previously shown how in-focus ptychography enables imaging at more than double the diffraction limit of the lens [3]. Here we also show how out-of-focus ptychography (Figure 1) improves the dose efficiency compared with ADF STEM (Figure 2a), simultaneously providing a fourtimes-faster acquisition, double the information limit and double the precision (Figure $2 b$ ).

By combining our design of electron microscope pixel array detector (EMPAD) [1] which has the dynamic range to record the complete distribution of transmitted electrons at every beam position, and a ptychographic phase retrieval algorithm to process the data, we have been able to increase the spatial resolution well beyond the traditional lens limitations reaching a $0.39 \AA$ resolution for $\mathrm{MoS}_{2}$, at the same dose and imaging conditions where conventional imaging modes reach only $0.98 \AA$ A.[3]

However, in-focus ptychography, like conventional STEM, is not well suited to imaging large areas at high spatial resolution - as the resolution is increased, the number of samples required grows quadratically in dwell time or dose. Operating out-of-focus decouples the resolution and real-space sampling requirements $[2,4,5]$, provided the detector has sufficient dynamic range and pixels. While simulations have shown the out-of-focus reconstructions to have better convergence at low dose, compare to in-focus [3], in practice this was not the case when simple initial probe estimates were used. With improved probe diversity, we have been able to image $120 \mathrm{~nm}$ fields of view with $0.69 \AA$ resolution at $80 \mathrm{keV}$ producing 6000x6000 pixel images. Figure 2 shows the dose dependence and resolution of out-of-focus ptychography on $\mathrm{WS}_{2}$ monolayers. Figure $2 \mathrm{~d}$ shows the precision with which we can measure $\mathrm{S}-\mathrm{S}$ or $\mathrm{W}$ $\mathrm{W}$ bond lengths, both in ptychography and ADF STEM, showing a roughly factor of two advantage for ptychography over ADF at the same dose.

The improved resolution, dose efficiency and robustness to environmental noise enabled by ptychography make it easy to identify defects such as sulfur monovacancies, as well as subtle structural arrangements and tilts on the sulfur sublattice that are undetectable by conventional imaging modes. [6] 

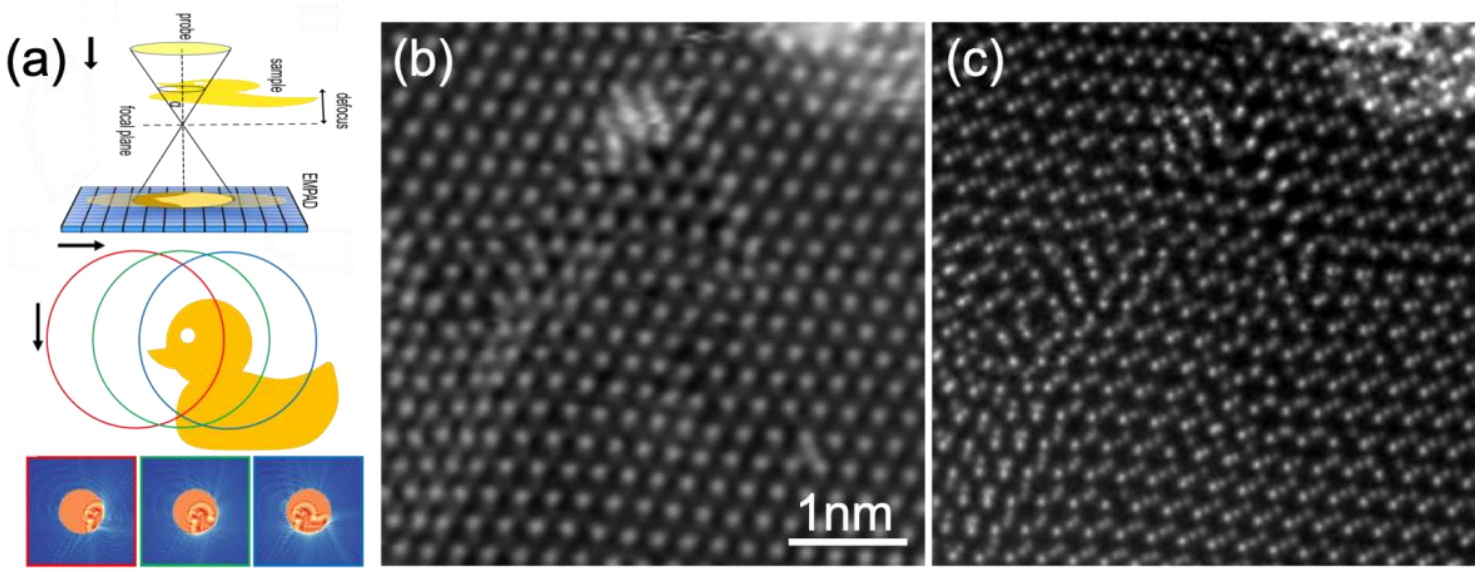

Figure 1. Performance of mixed-state, defocused ptychography demonstrated on corrugated MoS2 with macroscopic gaussian curvature. (a) Schematic of the defocus ptychography approach. At the bottom are 3 diffraction patterns simulated when the probe is illuminated at the red, green and blue positions respectively, which show the shadow images in the bright-field disk shifting accordingly with the probe position. The speckles in the dark-field region contain high frequency information needed for superresolution reconstructions. (b) ADF image from a grain boundary in curved MoS2. The contrast on the sulfur atoms is greatly reduced, with only the $\mathrm{W}$ remaining visible. (c) Defocused ptychographic reconstruction of the same area. In titled regions, the 2 sulfur atoms no longer lie atop each other and their projected separation gives the tilt angle of the three-dimensionally-curved sheet.
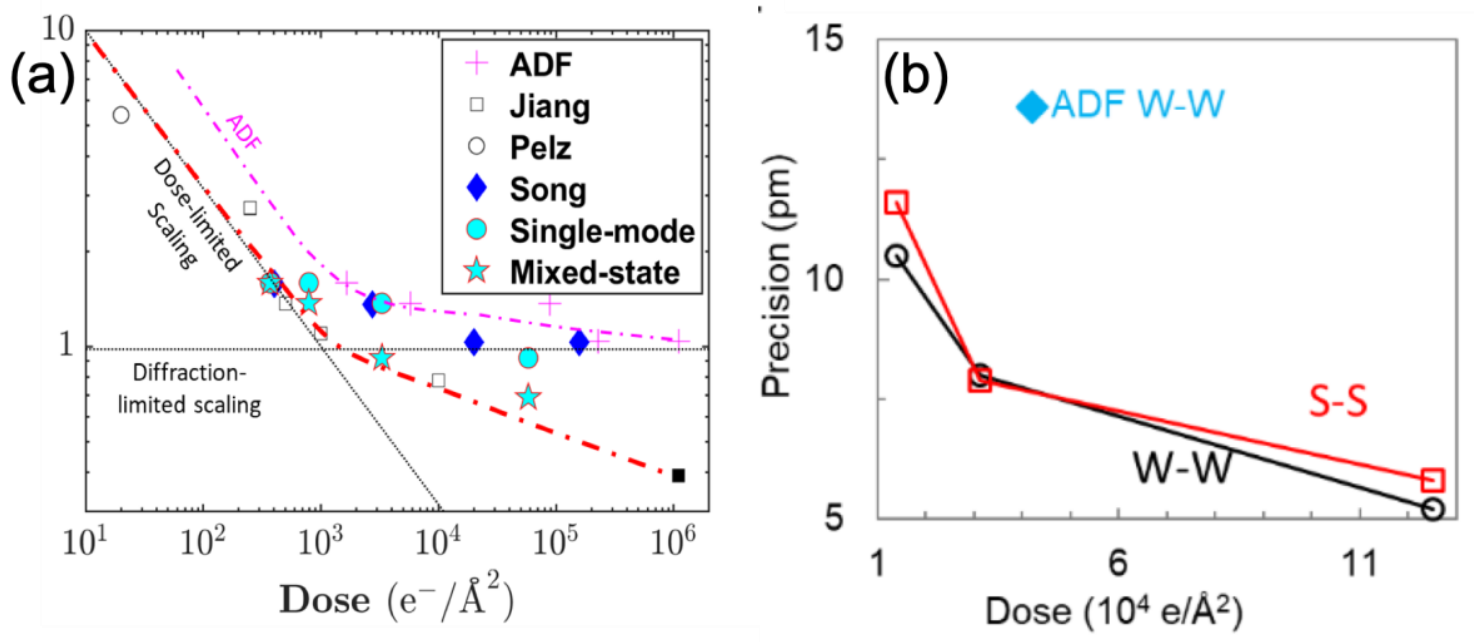

Figure 2. (a) Dose dependence of the information limit for low-angle ADF-STEM and electron ptychography for monolayer MoS2. Dose-limited resolution, which is inversely proportional to the square root of dose (linear in log-log plot), and aperture-limited resolution are indicated with dashed lines. Song and Pelz results from [4,5]. (b) Measurement precision of atomic distances between W-W and S-S in monolayer MoS2 as a function of the illumination dose. W-W bond distance precision from a conventional ADF image with a dose $4.2 \times 104$ e/Å2 denoted by a diamond symbol.

\section{References}

[1] Tate, M. W. et al. High dynamic range pixel array detector for scanning transmission electron microscopy. Microsc. Microanal. 22, 237-249 (2016). 
[2] A Maiden, et al, Ultramicroscopy 109 (2009), p. 1256.

[3] Jiang, Y. et al. Electron ptychography of 2D materials to deep sub-ångström resolution. Nature 559, 343-349 (2018).

[4] Pelz, P. M., Qiu, W. X., Bucker, R., Kassier, G. \& Miller, R. J. D. Low-dose cryo electron ptychography via non-convex Bayesian optimization. Sci. Rep. 7, 9883 (2017).

[5] Song, J. et al. Atomic Resolution Defocused Electron Ptychography at Low Dose with a Fast, Direct Electron Detector. Sci. Rep. 9, 3919 (2019).

[6] We thank Jiwoong Park, Saien Xie, Hui Gao, Ming-Hui Chiu, and Lain-Jong Li for samples. Research supported by US National Science Foundation (grants DMR-1539918, DMR-1719875, DMR-1429155). 